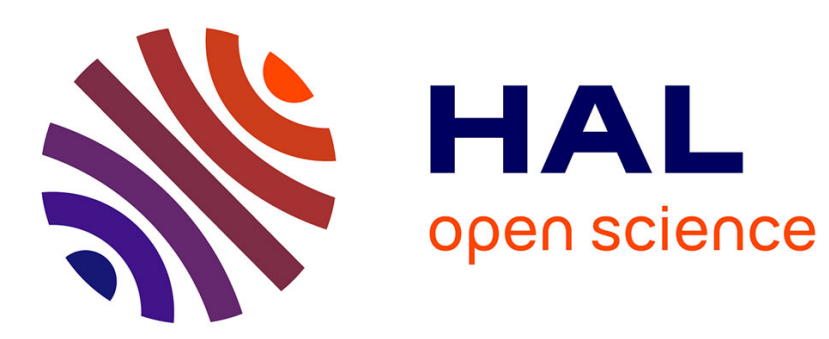

\title{
Rotationally averaged linear absorption spectra beyond the electric-dipole approximation
}

\author{
Nanna Holmgaard List, Trond Saue, Patrick Norman
}

\section{To cite this version:}

Nanna Holmgaard List, Trond Saue, Patrick Norman. Rotationally averaged linear absorption spectra beyond the electric-dipole approximation. Molecular Physics, 2017, 115 (1-2), pp.63-74. 10.1080/00268976.2016.1187773 . hal-01500621

\section{HAL Id: hal-01500621 \\ https://hal.science/hal-01500621}

Submitted on 31 Jan 2020

HAL is a multi-disciplinary open access archive for the deposit and dissemination of scientific research documents, whether they are published or not. The documents may come from teaching and research institutions in France or abroad, or from public or private research centers.
L'archive ouverte pluridisciplinaire HAL, est destinée au dépôt et à la diffusion de documents scientifiques de niveau recherche, publiés ou non, émanant des établissements d'enseignement et de recherche français ou étrangers, des laboratoires publics ou privés. 
To appear in Molecular Physics

Vol. 00, No. 00, Month 2009, 1-17

\title{
RESEARCH ARTICLE
}

\section{Rotationally averaged linear absorption spectra beyond the electric-dipole approximation}

\author{
Nanna Holmgaard List, ${ }^{a *}$ Trond Saue ${ }^{b}$ and Patrick Norman ${ }^{a \dagger}$ \\ ${ }^{a}$ Department of Physics, Chemistry and Biology, Linköping University, Linköping, SE \\ 58183, Sweden; ${ }^{b}$ Laboratoire de Chimie et Physique Quantiques, UMR 5626 - \\ CNRS/Université Toulouse III (Paul Sabatier), 118 route de Narbonne, F-31062 \\ Toulouse cedex, France \\ (Received 00 Month 200x; final version received 00 Month 200x)
}

\begin{abstract}
Based on the recently developed implementation of the full semi-classical field-matter interaction operator [List et al. in J. Chem. Phys. 142, 244111 (2015)], we present a numerically accurate yet efficient scheme to perform rotational averaging of linear absorption spectra beyond the electric-dipole approximation. This allows for a gauge-origin independent determination of UV/vis and X-ray absorption spectra for randomly oriented systems such as multilayers, liquids, and gas phase samples. The approach is illustrated by the determination of spectral intensities of electric-dipole allowed $\pi \rightarrow \pi^{*}$ transitions and electric-dipole forbidden $n \rightarrow \pi^{*}$ transitions in the UV-vis region of the spectrum as well as electric-dipole forbidden $1 s \rightarrow 3 d$ transitions in the X-ray region of the spectrum. The employed Lebedev quadrature scheme shows very fast convergence with respect to the number of symmetry-independent quadrature points - in all considered cases, the oscillator strengths for the randomly oriented systems are fully converged with use of only seven quadrature points.
\end{abstract}

Keywords: response theory, multipole-expansion free, rotational averaging, Lebedev quadrature, X-ray absorption

\section{Introduction}

Light absorption and emission as due to the interaction between electromagnetic field radiation and molecular compounds are fundamental and essential ingredients in Nature as well as every-day life. Notable examples are given in terms of the conversion of solar energy by means of photosynthesis in green plants or, more broadly, the entire scientific field of photochemistry; [1] chromphores and dyes that give rise to a spectrum of colors in liquids, pigments, and molecular materials; the production of light in chemiluminescence and bioluminescence; [2] artificial light harvesting and organic solar cells or, more broadly, large parts of the scientific field of photonics; [3] and, not least, the plethora of spectroscopies that we have designed to probe and investigate the physical and chemical properties of molecules and molecular materials in various environments and phases. [4] We also design photoactive probes to selectively target host systems as to get indirect information of the host rather than the probe itself, as exemplified by the development of palettes of luminescent conjugated oligothiophenes for the detection and identification of protein aggregates.[5]

\footnotetext{
*Corresponding author. Email: nanna.h.list@gmail.com
}

${ }^{\dagger}$ Email: panor@ifm.liu.se 
In all of the phenomena, applications, and scientific fields mentioned above, the scientific community leverages the fundamental microscopic understanding obtained by means of computational chemistry to achieve rational molecular design and material performance enhancements. It comes therefore as no surprise that theoretical chemists, and in particular quantum chemists, have dedicated large efforts to develop methods and algorithms to accurately yet efficiently include the effects of external time-dependent electromagnetic fields on the molecular charge density. Most notably, there exist today several basic formulations of response theory with algorithmic and program implementations for virtually all standard electronic structure theory methods on the market.[6] In all of these instances, a semi-classical description is employed for the external field and it is common to assume that the field is sufficiently weak for perturbation theory to be applicable.[79] Thereafter differences start to appear, however, as one introduces the coupling terms between the semi-classical fields and the quantum mechanical system into the Hamiltonian. Choices are made based on the level of accuracy needed, algorithmic ease, and computational speeds. In absorption and emission studies, the component of the magnetic field can often be ignored as its effect on the molecular charges is weaker than electric fields by a factor of $1 / c$. If, on the other hand, a natural dichroism in isotropic samples is studied, then it is the mere presence of the magnetic component of the radiation field that gives rise to a differential absorption by the sample.

In this context, we have recently investigated the use of an exact and complete inclusion of electromagnetic fields in the interaction Hamiltonian by means of the principle of minimal coupling.[10] The assumptions made in the implementation of this strategy involved the specification of a state of linear polarization and a direction of propagation of a harmonic plane wave. Since any form of multipole expansion was avoided, response functions involving this full field-matter interaction operator are intrinsically gauge-origin invariant, and our formulation thus leads to physical observables that are independent of the gauge origin. The contributions to both absorptive and dispersive parts of response functions from all higher-order combinations of multipole moment operators are included in this treatment, and for instance natural dichroism is directly accounted for in the absorptive part of the linear response function.

For studies of oriented samples, such as the calculation of a near-edge X-ray absorption fine structure (NEXAFS) spectrum of a self-assembled monolayer at normal or grazing incidence,[11] the scheme presented by List et al.[10] is well suited. However, for multilayer samples, liquids, or otherwise randomly oriented systems, there is a need to perform spectrum averaging over molecular orientations in the laboratory frame of reference, or, equivalently, averaging over light propagation and polarization directions in the molecular frame. The advantage of performing a multipole moment expansion of the interaction operator becomes obvious at this point in the calculation, since, for every given order in the expansion, the orientational average of the corresponding property tensor can be performed analytically $[8,12-17]$ - e.g. the orientational average of the electric-dipole polarizability is referred to as the isotropic polarizability and is equal to a simple tensor trace. The same approach cannot be taken in our case, but, as will be demonstrated in the present work, it is possible to introduce the numerical quadrature integration schemes by Lebedev and co-workers[18-23] to achieve efficient and numerically accurate spectrum calculations for randomly oriented samples.

In the following, we will present a scheme for rotational averaging of linear absorption cross sections beyond the electric-dipole (ED) approximation associated with the interaction of a molecule with a linearly polarized electromagnetic field. 
We will refer to results obtained at this level of theory by the label BED and contrast these with the corresponding results obtained in the ED approximation. The remainder of this work is organized as follows: In Section 2, we give a brief recapitulation of the theoretical background relevant for the description of linear absorption in the BED framework as well as present a numerical scheme for the determination of spectra for randomly oriented samples. Computational details are given in Section 3. Representative numerical illustrations of the convergence behavior of the proposed scheme are presented in Section 4 by example calculations of UV/vis and X-ray absorption spectra of formaldehyde and acrolein and X-ray absorption spectra of ferrocene and vinylferrocene.

\section{Theory}

We will consider a collection of randomly oriented, identical, non-interacting closed-shell molecules subjected to a weak, monochromatic, linearly polarized electromagnetic field oscillating at an angular frequency $\omega$. The interaction between the molecular sample and the external field will be treated in the commonly used semi-classical framework, where the incident radiation is described as a classical field in terms of auxiliary scalar and vector potentials, $\phi(\boldsymbol{r}, t)$ and $\boldsymbol{A}(\boldsymbol{r}, t)$. Using the minimal coupling formulation of the interaction, the field-matter interaction operator can in the Coulomb gauge $(\nabla \cdot \boldsymbol{A}=0$, choosing $\phi=0)[24]$ be written as

$$
\hat{V}(t)=\frac{e}{m_{e}} \sum_{i=1}^{N} \boldsymbol{A}\left(\boldsymbol{r}_{i}, t\right) \cdot \hat{\boldsymbol{p}}_{i}=\sum_{ \pm \omega} \hat{V}^{\omega} e^{-i \omega t}
$$

with Fourier components given by

$$
\hat{V}^{ \pm \omega}= \pm \sum_{i=1}^{N} \frac{e \mathcal{E}_{0}}{2 i \omega m_{e}} e^{ \pm i \boldsymbol{k} \cdot \boldsymbol{r}_{i}}\left(\boldsymbol{\epsilon} \cdot \hat{\boldsymbol{p}}_{i}\right)
$$

In writing Eq. (1), we have neglected the term quadratic in $\boldsymbol{A}$, which does not contribute to linear absorption.[10] The linear momentum operator is given by $\hat{\boldsymbol{p}}_{i}=-i \hbar \nabla_{i}, \hbar$ is the reduced Planck constant, $-e$ and $m_{e}$ are the charge and mass of an electron, respectively, and $N$ is the total number electrons in the molecule. $\mathcal{E}_{0}$ is the real-valued external field amplitude, and $\boldsymbol{k}$ is the wave vector that describes the propagation direction of the light beam with norm $k=|\boldsymbol{k}|=\omega / c_{0}$, where $c_{0}$ is the speed of light in vacuo. Finally, $\boldsymbol{\epsilon}$ is a unit vector perpendicular to $\boldsymbol{k}$, specifying the polarization direction of the light.

\subsection{Linear absorption of linearly polarized light}

In this section, we will review the theory necessary for describing linear absorption using the field-matter interaction operator in Eq. (1). Following the energy-loss approach, molecular absorption cross sections, describing the rate of energy flow from an external electromagnetic field to a molecule, can be related to resonanceconvergent complex molecular response functions. $[8,25]$ In particular, the linear absorption cross section, $[10]$

$$
\sigma(\omega)=\frac{4 \omega}{\varepsilon_{0} c_{0} \mathcal{E}_{0}^{2}} \operatorname{Im}\left[G_{\hat{\epsilon}}^{\hat{k}}(-\omega ; \omega)\right]
$$


is proportional to the imaginary part of the general molecular polarizability $G_{\hat{\epsilon}}^{\hat{k}}(-\omega ; \omega)$ that represents the linear response of the molecule to a linearly polarized field characterized by polarization and propagation directions $(\hat{\epsilon}, \hat{k})$. In exact-state theory, the complex polarizability can be expressed as a sum over excited states according to

$$
G_{\hat{\epsilon}}^{\hat{k}}(-\omega ; \omega)=\frac{1}{\hbar} \sum_{n>0}\left[\frac{\left\langle 0\left|\hat{V}^{-\omega}\right| n\right\rangle\left\langle n\left|\hat{V}^{\omega}\right| 0\right\rangle}{\omega_{n 0}-\omega-i \gamma_{n 0}}+\frac{\left\langle 0\left|\hat{V}^{\omega}\right| n\right\rangle\left\langle n\left|\hat{V}^{-\omega}\right| 0\right\rangle}{\omega_{n 0}+\omega+i \gamma_{n 0}}\right],
$$

where $\hbar \omega_{n 0}$ is the transition energy from the electronic ground state $|0\rangle$ to the electronic excited state $|n\rangle$. The phenomenological damping parameters $\gamma_{n 0}=$ $\left(2 \tau_{n}\right)^{-1}$ are related to the lifetimes $\tau_{n}$ of the exited states, assuming exponential decay. In approximate-state theory, the resonance-convergent polarizability can be addressed in the framework of the complex polarization propagator (CPP) approach. [26-28] Eq. (4) is then replaced by a matrix equation in which the explicit reference to the excited states is avoided.[10] In other words, the linear absorption cross section can be evaluated without having to resolve the individual eigenvectors of the electronic Hessian. This is particularly important because it makes it possible to treat core excitations without imposing restrictions on the excitation manifold.

In the alternative and traditional approach, one considers the infinite-lifetime limit $\left(\gamma_{n 0}=0\right)$ of the linear absorption cross section in Eq. (4). Focusing on the absorptive part of the response function, we obtain[29, 30]

$$
\lim _{\gamma \rightarrow 0} \sigma(\omega)=\frac{4 \pi^{2} \alpha}{e^{2} \omega} \sum_{n>0}\left[\delta\left(\omega-\omega_{n 0}\right)\left|\mathrm{TM}_{\mathrm{BED}}^{n 0}\right|^{2}\right]
$$

where $\alpha=e^{2} /\left(4 \pi \varepsilon_{0} \hbar c_{0}\right)$ is the dimensionless fine structure constant and $\mathrm{TM}_{\mathrm{BED}}^{n 0}$ the transition moment associated with the $0 \rightarrow n$ excitation

$$
\mathrm{TM}_{\mathrm{BED}}^{n 0}=\frac{e}{m_{e}}\left\langle 0\left|\sum_{i=1}^{N} e^{-i \boldsymbol{k} \cdot \boldsymbol{r}_{i}}\left(\boldsymbol{\epsilon} \cdot \hat{\boldsymbol{p}}_{i}\right)\right| n\right\rangle .
$$

Integration over frequencies of the right hand side of Eq. (5) leads to a sum of terms

$$
\sigma_{n 0}=\frac{\pi^{2} \hbar e^{2}}{\left(4 \pi \varepsilon_{0}\right) m_{e} c_{0}} f_{n 0}
$$

where appears the dimensionless oscillator strength, $[24,30]$

$$
f_{n 0}=\frac{2 m_{e}}{e^{2} \hbar \omega_{n 0}}\left|\mathrm{TM}_{\mathrm{BED}}^{n 0}\right|^{2},
$$

which represents the relative intensity of absorption with respect to a harmonic oscillator model.

Eqs. (4) and (8) are typically simplified by invoking a Taylor expansion of the exponential operator in orders of the wave vector $\boldsymbol{k}, i . e ., e^{i \boldsymbol{k} \cdot \boldsymbol{r}}=1+i \boldsymbol{k} \cdot \boldsymbol{r}-\frac{1}{2}(\boldsymbol{k} \cdot \boldsymbol{r})^{2}+$ $\cdots$. Retaining only the zeroth-order term defines the ED approximation, which relies on the assumption that the wavelength of the electromagnetic radiation field is much larger than the molecular dimension $(k r \ll 1)$. In most cases, this is a good approximation for transitions in the $\mathrm{UV} /$ vis region, where the field indeed appears 
uniform across the molecule. In X-ray spectroscopies, the short wavelength of the radiation becomes comparable to the spatial extension of the probed molecular systems, and intuitively, this would put the use of the ED approximation in doubt. Recently, we have demonstrated the validity of the ED approximation for ED allowed transitions in $K$-edge X-ray absorption spectroscopy. It was shown that the otherwise short wavelengths are compensated by the spatial compactness of the initial $1 s$-orbitals, and that the $k r$ value remains below unity for all elements in the periodic table.[10] However, when probing excitations that are ED forbidden by symmetry reasons, or when considering more complex physical phenomena, the higher-order contributions accounting for the spatial variations of the field need to be taken into account. A prominent example is pre-edge features in metal $K$-edge $\mathrm{X}$-ray absorption spectra of transition metal complexes, which are often governed by ED forbidden transitions from metal $1 s$ - to $d$-orbitals. [31-33]

An important aspect of going beyond the ED approximation by including additional terms in the multipole expansion of the physical observable is that the individual terms, beyond the lowest non-zero multipole order, are origindependent.[8, 34] Accordingly, special attention has to be paid to ensure origininvariance of the physical observables when using a truncated multipole expansion formulation. [35-37] In particular, Bernadotte et al. showed that origin-independent oscillator strengths can be obtained by including all terms in the oscillator strength consistently through a given order in the wave vector. [37] However, the cross sections introduced in this origin-independent but truncated multipole expansion formulation (see Eq. 62, Ref. [37]) can in certain cases result in unphysical, negative oscillator strengths, as recently reported in Ref. [38].

In this work, we take as starting point for the rotational averaging of the linear absorption cross section the untruncated expressions in Eqs. (4) and (8) involving the complete field-matter interaction operator. Using the exact semi-classical representation will, by necessity, lead to rotationally averaged cross sections that are origin-independent and always positive, as they should be.

\subsection{Rotational Averaging}

In Eqs. (4) and (8), we assumed that the components of $(\boldsymbol{k}, \boldsymbol{\epsilon})$ and $\left(\boldsymbol{r}_{i}, \hat{\boldsymbol{p}}_{i}\right)$ refer to a common coordinate system, say a space-fixed frame with unit vectors $\left\{\mathbf{e}_{X}, \mathbf{e}_{Y}, \mathbf{e}_{Z}\right\}$. For convenience, the space-fixed coordinate system is defined such that the $Z$-axis is parallel to the propagation direction and the $X$-axis to the polarization vector, i.e., $\boldsymbol{k}=k \mathbf{e}_{Z}$ and $\boldsymbol{\epsilon}=\mathbf{e}_{X}$. This corresponds to an experimental setup, where the absorbing molecules are oriented in a specific way with respect to the incoming electromagnetic radiation. In the majority of experiments, the molecules in the sample are free to rotate, and the theory as described above stands in need of further refinement before its results can be related to experimentally measured absorption cross sections. Under these circumstances, it is necessary to perform rotational averages of Eqs. (4) and (8) to account for the distribution of molecular orientations in the sample. For simplicity, we shall assume an isotropic distribution of molecules in which case the problem reduces to averaging over all possible orientations of the molecule relative to the propagation and polarization directions.

We therefore consider a molecule-fixed coordinate frame with unit vectors $\left\{\mathbf{e}_{x}, \mathbf{e}_{y}, \mathbf{e}_{z}\right\}$. In this frame, the coordinates $\boldsymbol{r}^{\prime \prime}$ are related to those of the spacefixed frame, $\boldsymbol{r}$, by

$$
\boldsymbol{r}^{\prime \prime}=\mathbf{R}\left(\phi, \mathbf{e}_{Z}\right) \mathbf{R}\left(\theta, \mathbf{e}_{Y}\right) \mathbf{R}\left(\chi, \mathbf{e}_{Z}\right) \boldsymbol{r}
$$


where the total rotation is expressed in terms of the three Euler angles $(\phi, \theta, \chi)[39]$

$\mathbf{R}\left(\phi, \mathbf{e}_{Z}\right) \mathbf{R}\left(\theta, \mathbf{e}_{Y}\right) \mathbf{R}\left(\chi, \mathbf{e}_{Z}\right)=\left(\begin{array}{ccc}\cos \phi & -\sin \phi & 0 \\ \sin \phi & \cos \phi & 0 \\ 0 & 0 & 1\end{array}\right)\left(\begin{array}{ccc}\cos \theta & 0 & \sin \theta \\ 0 & 1 & 0 \\ -\sin \theta & 0 & \cos \theta\end{array}\right)\left(\begin{array}{ccc}\cos \chi & -\sin \chi & 0 \\ \sin \chi & \cos \chi & 0 \\ 0 & 0 & 1\end{array}\right)$

In the molecule-fixed frame, the wave and polarization vectors are given by

$$
\boldsymbol{k}=k \mathbf{e}_{r} ; \quad \boldsymbol{\epsilon}=\cos \chi \mathbf{e}_{\theta}+\sin \chi \mathbf{e}_{\phi}
$$

in terms of unit vectors

$$
\begin{aligned}
\mathbf{e}_{\theta} & =\mathbf{e}_{x} \cos \theta \cos \phi+\mathbf{e}_{y} \cos \theta \sin \phi-\mathbf{e}_{z} \sin \theta \\
\mathbf{e}_{\phi} & =-\mathbf{e}_{x} \sin \phi+\mathbf{e}_{y} \cos \phi \\
\mathbf{e}_{r} & =\mathbf{e}_{x} \sin \theta \cos \phi+\mathbf{e}_{y} \sin \theta \sin \phi+\mathbf{e}_{z} \cos \theta .
\end{aligned}
$$

The isotropic average of the oscillator strength, or cross section, over the three Euler angles is defined as

$$
\left\langle f_{n 0}\right\rangle_{\phi, \theta, \chi}=\frac{1}{8 \pi^{2}} \int_{0}^{2 \pi} \mathrm{d} \phi \int_{0}^{\pi} \sin \theta \mathrm{d} \theta \int_{0}^{2 \pi} f_{n 0} \mathrm{~d} \chi
$$

We note that in the space-fixed frame the dependence on the Euler angles enters parametrically in the electronic wave functions describing the ground state $|0\rangle$ and the excited state $|n\rangle$, whereas in the molecule-fixed frame all dependence is in the wave and polarization vectors in the field-matter interaction operator.

We now introduce an intermediate frame whose coordinates are given by

$$
\boldsymbol{r}^{\prime}=\mathbf{R}\left(\chi, \mathbf{e}_{Z}\right) \boldsymbol{r}=\mathbf{R}\left(-\theta, \mathbf{e}_{Y}\right) \mathbf{R}\left(-\phi, \mathbf{e}_{Z}\right) \boldsymbol{r}^{\prime \prime}
$$

In this frame, the representation of the wave and polarization vectors are given by

$$
\boldsymbol{k}^{\prime}=(0,0, k) ; \quad \boldsymbol{\epsilon}^{\prime}=(\cos \chi, \sin \chi, 0)
$$

In the intermediate frame, the dependence on the two Euler angles $(\theta, \phi)$ is in the electronic wave functions, whereas the dependence on the Euler angle $\chi$ only enters the polarization vector. The averaging over the latter angle can now be performed straightforwardly in an analytical manner. It reduces to

$$
\left\langle f_{n 0}\right\rangle_{\chi}=\frac{2 m_{e}}{e^{2} \hbar \omega_{n 0}}\left(\frac{e}{m_{e}}\right)^{2}\left\langle\epsilon_{\alpha}^{\prime} \epsilon_{\beta}^{\prime}\right\rangle_{\chi}\left\langle 0\left|\sum_{i=1}^{N} e^{-i k z_{i}^{\prime}} \hat{p}_{i \alpha}^{\prime}\right| n\right\rangle\left\langle n\left|\sum_{j=1}^{N} e^{+i k z_{j}^{\prime}} \hat{p}_{j \beta}^{\prime}\right| 0\right\rangle
$$

where repeated Greek indices indicate summation over Cartesian coordinates. The only non-zero elements are

$$
\left\langle\epsilon_{x}^{\prime} \epsilon_{x}^{\prime}\right\rangle_{\chi}=\left\langle\epsilon_{y}^{\prime} \epsilon_{y}^{\prime}\right\rangle_{\chi}=\frac{1}{2}
$$


such that the final expression for the $\chi$-averaged oscillator strength reads

$$
\left\langle f_{n 0}\right\rangle_{\chi}=\frac{m_{e}}{e^{2} \hbar \omega_{n 0}}\left(\frac{e}{m_{e}}\right)^{2} \sum_{\alpha=x, y}\left|\left\langle 0\left|\sum_{i=1}^{N} e^{-i k z_{i}^{\prime}} \hat{p}_{i \alpha}^{\prime}\right| n\right\rangle\right|^{2} .
$$

In a similar manner, the $\chi$-averaged linear absorption cross section becomes

$$
\langle\sigma(\omega)\rangle_{\chi}=\frac{2 \omega}{\varepsilon_{0} c_{0} \mathcal{E}_{0}^{2}}\left(\operatorname{Im}\left[G_{x^{\prime}}^{z^{\prime}}(-\omega ; \omega)+\operatorname{Im}\left[G_{y^{\prime}}^{z^{\prime}}(-\omega ; \omega)\right]\right) .\right.
$$

The $\chi$-averaged quantities Eqs. (17) and (18) depend on the solid angle $(\theta, \phi)$, which specifies the orientation of the molecule with respect to the propagation direction. The isotropic averaging of the linear absorption has therefore been reduced to a two-angle integration of Eqs. (17) and (18) over the solid angle. To get a flavor of how the averaging work, we consider the average of the oscillator strength within the ED approximation

$$
\left\langle f_{n 0}^{\mathrm{ED}}\right\rangle_{\phi, \theta, \chi}=\frac{m_{e}}{e^{2} \hbar \omega_{n 0}}\left(\frac{e}{m_{e}}\right)^{2}\left\langle\sum_{\alpha=x, y}\left|\left\langle 0\left|\sum_{i=1}^{N} \hat{p}_{i \alpha}^{\prime}\right| n\right\rangle\right|^{2}\right\rangle_{\phi, \theta, \chi} .
$$

The average over the final two angles $(\theta, \phi)$ is best done in the molecule-fixed frame. Inserting the relations

$$
\begin{aligned}
& \hat{p}_{i x}^{\prime}=\cos \theta \cos \phi \hat{p}_{i x}^{\prime \prime}+\cos \theta \sin \phi \hat{p}_{i y}^{\prime \prime}-\sin \theta \hat{p}_{i z}^{\prime \prime} \\
& \hat{p}_{i y}^{\prime}=-\sin \phi \hat{p}_{i x}^{\prime \prime}+\cos \phi \hat{p}_{i y}^{\prime \prime},
\end{aligned}
$$

and performing the average gives the well-known expression for the isotropically averaged oscillator strength in the velocity representation

$$
\left\langle f_{n 0}^{\mathrm{ED}}\right\rangle_{\phi, \theta, \chi}=\frac{2}{3 m_{e} \hbar \omega_{n 0}} \sum_{\alpha=x, y, z}\left|\left\langle 0\left|\sum_{i=1}^{N} \hat{p}_{i \alpha}^{\prime \prime}\right| n\right\rangle\right|^{2} .
$$

Since it effectively amounts to a matrix trace, it is valid in all frames considered. In the general case, the $(\theta, \phi)$-integration will be performed numerically using the schemes of Lebedev and co-workers.[18-23] The distribution of quadrature points over a unit sphere for a given Lebedev grid defines the propagation directions included in the numerical integration, and the $\chi$-average is performed in accordance with Eqs. (17) and (18) by considering two orthogonal polarization directions for each wave vector, forming a right-handed coordinate system. The Lebedev grids have been constructed under octahedral symmetry, which makes them suited for the present context. In particular, the $\chi$-averaged transition strength is identical for quadrature points corresponding to $\boldsymbol{k}$ and $\boldsymbol{- k}$. This can be exploited to limit the $(\theta, \phi)$-integration to a hemisphere, thereby reducing the number of quadrature points needed for a given Lebedev grid by a factor of two in the general case. Additional savings can be achieved for symmetric molecules where the calculations may be restricted to those quadrature points that are not related by symmetry operations in the given point group. 


\section{Computational Details}

The lowest energy valence and carbon $K$-edge excitations in formaldehyde and trans-acrolein have been used to study the convergence behavior of the proposed Lebedev-based scheme for obtaining rotationally averaged linear absorption cross sections involving the full field-matter interaction operator. The molecular geometries were optimized under the constraint of $C_{s}$ and $C_{2 v}$ symmetry, respectively, at the DFT level of theory using the B3LYP[40-43] exchange-correlation functional and the cc-pVTZ[44] basis set. The property calculations for formaldehyde and acrolein were performed using the CAM-B3LYP $100 \%$ functional, designed for the purpose of describing core electron excitations. [28] This is a modified version of the Coulomb-attenuated method CAM-B3LYP[45] functional taking into account $100 \%$ Hartree-Fock Coulombic interactions in the long-range limit $(\alpha=0.19$, $\beta=0.81$, and $\mu=0.33 a_{0}^{-1}$ ). For the calculation of oscillator strengths, we have employed the restricted-channel approximation.[28, 46] Specifically, the excitation manifold in the linear response calculation of formaldehyde and acrolein was restricted to excitations originating from the carbon $1 s$-orbitals to any of the virtual orbitals to selectively target the $\mathrm{C} K$-edge. Furthermore, we have considered the Fe $K$-edge NEXAFS spectrum of ferrocene (iron bis-cyclopentadienyl) and vinylferrocene (ferrocene with a vinyl substituent at one of the cyclopentadienyl rings) to illustrate the applicability of the presented procedure for probing quadrupole intensities of isotropic samples. The structures of ferrocene and vinylferrocene in eclipsed conformation have been taken from Ref. [47], where they were optimized using the BP86 functional and the TZP basis set. The frequency-dependent absorption cross sections, or, in other words, the linear absorption spectra, of the ferrocene derivatives were determined at the B3LYP level within the CPP formalism using the most recent $\mathrm{CPP}$ algorithm[48, 49] and a lifetime broadening $\gamma=1000 \mathrm{~cm}^{-1}$ (or $0.1240 \mathrm{eV}$ ). It is well-known that the energy separation between the pre-edge peaks in substituted ferrocenes is highly sensitive to the choice of functional, and that it is exaggerated by B3LYP. $[47,50]$ We primarily adopt the use of this functional for the ferrocene derivatives to be able to compare our results with those of previous work. The $(\theta, \phi)$-angular integration of the linear absorption cross section was handled by a set of highly accurate Lebedev grids. $[18,51]$ All property calculations employed the aug-cc-pVDZ[44, 52, 53] basis set and were performed with a locally modified version of the Dalton program. [54, 55]

\section{Results and Discussion}

To investigate the convergence of the numerical scheme presented above for the calculation of rotationally averaged linear absorption cross sections using the full field-matter interaction operator, we consider a set of prototypical transitions in the UV/vis and X-ray spectral regions. Specifically, we consider the lowest $n \rightarrow \pi^{*}$ excitations in formaldehyde and acrolein, the $\pi \rightarrow \pi^{*}$ transition in acrolein, $\mathrm{C} 1 s \rightarrow$ $\pi^{*}$ transitions in formaldehyde and acrolein, and mixes of Fe $1 s \rightarrow 3 d$ and $1 s \rightarrow 4 p$ transitions in ferrocene and vinylferrocene (see Fig. 1 for chemical structures and atom labeling used for the $1 s \rightarrow \pi^{*}$ transitions in acrolein). These include ED allowed and forbidden transitions in both spectral regions, and this allows us to study how the nature of the electronic transitions, and thus the importance of BED effects, influences the numerical convergence of the adopted quadrature scheme used to determine rotationally averaged oscillator strengths. It is instructive to start by considering the behavior of the $\chi$-averaged linear absorption cross section, or oscillator strength, to be numerically integrated with respect to the direction of 


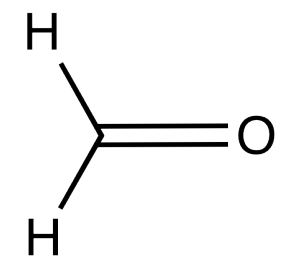

(a)

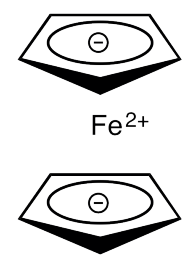

(c)
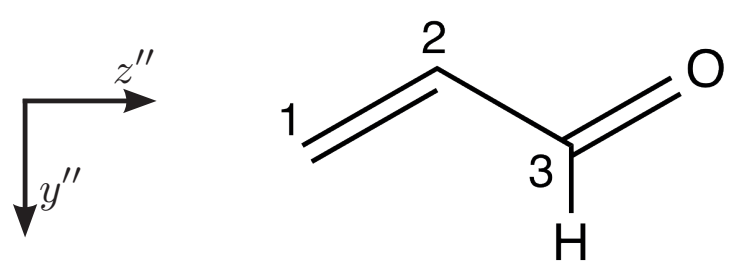

(b)

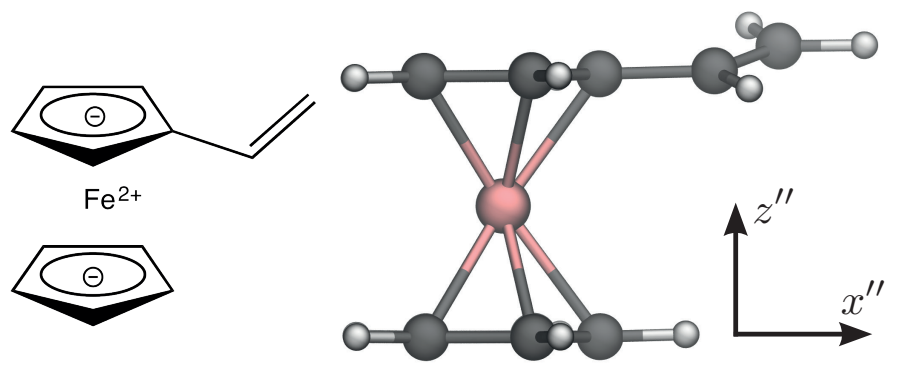

(d)

Figure 1. Chemical structures of the molecules considered: (a) formaldehyde; (b) acrolein; eclipsed conformation of (c) ferrocene and (d) vinylferrocene. The orientation of the molecules within the molecule-fixed coordinate system is also shown.

the wave vector $\boldsymbol{k}$ over a unit sphere. Note that the averaging over $\chi$ means that the information about polarization dependence is lost.

\subsection{Formaldehyde and acrolein}

First, we consider the lowest valence and $\mathrm{C} K$-edge transitions in the two aldehydes, formaldehyde and acrolein. The excitation energies as calculated at the CAM-B3LYP ${ }_{100 \%}$ /aug-cc-pVDZ of theory are given in the upper part of Table 1, and the $(\theta, \phi)$-dependency of the associated $\chi$-averaged oscillator strength $\langle f\rangle_{\chi}$ for a representative set of the considered transitions is illustrated in Figs. 2 and 3 for acrolein and formaldehyde, respectively. To dissect the impact of going beyond the ED approximation, we include in Fig. 2 (bottom row) also the difference $\Delta\langle f\rangle_{\chi}$ between the oscillator strengths obtained beyond and within the ED approximation. As a general comment, we note that the $\chi$-averaged oscillator strength is seen to be identical for wave vectors $\boldsymbol{k}$ and $\boldsymbol{- k}$, which we exploit in the Lebedev quadrature.

As demonstrated in Ref. [10], the ED approximation is useful for describing linear absorption of ED allowed excitations across the entire frequency range, and BED corrections are expected to be significant only for ED forbidden transitions. In formaldehyde, the $n \rightarrow \pi^{*}$ transition is strictly ED forbidden by symmetry the excited state spans the $A_{2}$ irreducible representation of the $C_{2 v}$ point groupwhereas in acrolein, the corresponding transition is ED allowed, and the extent to which it may be observable in the ED approximation depends sensitively on the local symmetry of the carbonyl group.

There is a high degree of directional dependence in the linear absorption cross section for these planar systems. The $\pi \rightarrow \pi^{*}$ transitions are most effectively 
(a)

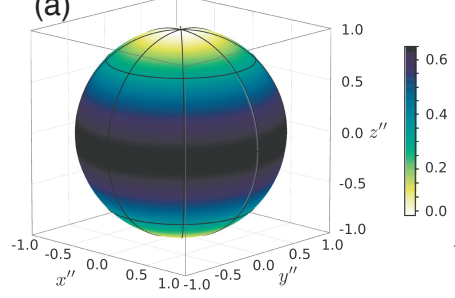

(b)

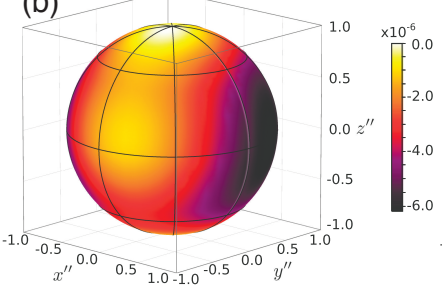

(c)

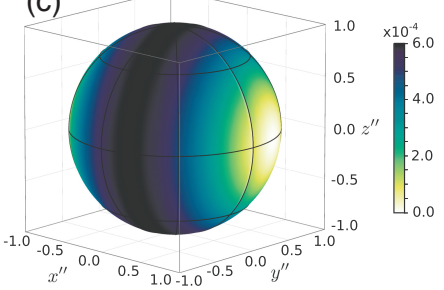

(d)

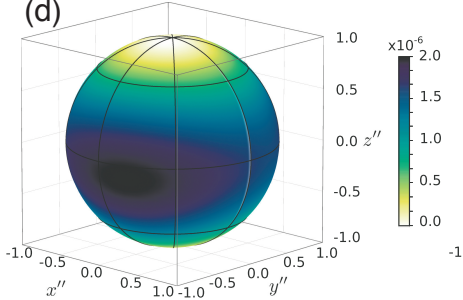

(e)

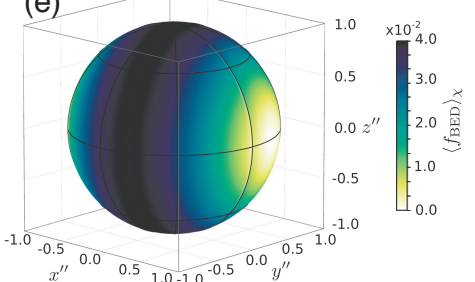

(f)

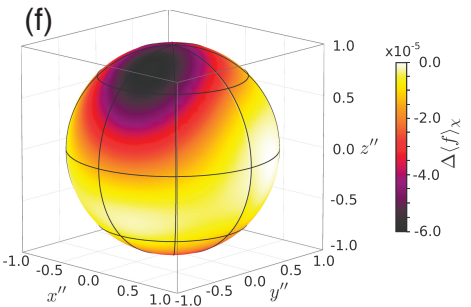

Figure 2. $(\theta, \phi)$-dependency of the $\chi$-averaged oscillator strength $\left\langle f_{\mathrm{BED}}\right\rangle_{\chi}$ (top row) and the corresponding differences $\Delta\langle f\rangle_{\chi}$ between BED and ED (bottom row) for selected transitions in acrolein: (a,b) the lowest $\pi \rightarrow \pi^{*}$ transition $(\mathrm{c}, \mathrm{d})$ the lowest $n \rightarrow \pi^{*}$ transition and (e,f) the $\mathrm{C}_{1} 1 s \rightarrow \pi^{*}$ transition. The orientation of acrolein in the molecule-fixed frame as well as the atom labeling is given in Fig. 1.

induced by an electric field directed along the conjugation axis (the $z$-direction in Fig. 1), and thus propagating in a direction orthogonal to the conjugation axis, whereas zero absorption is associated with fields propagating along the conjugation axis. This is illustrated in Fig. $2 \mathrm{a}$ in terms of the $\chi$-averaged oscillator strength for the $\pi \rightarrow \pi^{*}$ transition in acrolein. The nearly perfect cylindrical symmetry about the $z$-axis reflects the fact that the main component of the leading ED contribution is invariant to the direction of propagation orthogonal to the conjugation axis (within the $x y$-plane). The effects of going beyond the ED approximation are shown in Fig. 2b. Note that $\Delta\langle f\rangle_{\chi}$ is negative and negligible in all directions of light propagation, as expected for BED corrections to ED allowed transitions.[10] Similar observations can be made for the ED allowed $\mathrm{C} K$-edge transitions in both aldehydes. This is exemplified in Figs. $2 \mathrm{e}$ and $2 \mathrm{f}$ for the $\mathrm{C}_{1} 1 s \rightarrow \pi^{*}$ transition in acrolein. In this case, the directional dependence reflects the $\pi^{*}$-orbital entirely, and thus the cylindrical symmetry is about the $x$-axis. The BED effects for the core transitions are two orders of magnitude larger than for the valence $\pi \rightarrow \pi^{*}$ excitation in acrolein, but they are still negligible (0.006-0.07\%) for all practical purposes.

For the strictly ED forbidden $n \rightarrow \pi^{*}$ transition in formaldehyde, the $\chi$-averaged oscillator strength displays a more complex dependence on the direction of light propagation, see Fig. 3. In this case, the leading contributions stem from the terms involving the magnetic-dipole and electric-quadrupole operators. As a consequence, with formaldehyde placed in the $y z$-plane and the $\mathrm{C}=\mathrm{O}$ bond along the $z$-axis in the molecular frame, the strongest absorption intensity will be due to light propagating in the $x$-direction with an electric-field polarization along $y$, and vice versa. Inspection of the individual lowest-order contributions (data not shown) reveals that the magnetic-dipole term is two orders of magnitude larger than the electricquadrupole contribution (see further discussion in Section 4.3). Note that this division into magnetic-dipole and electric-quadrupole contributions is here unambiguous since, in the present case, the vanishing ED oscillator strength implies gauge-origin invariance for these terms. In analogy to the ED term for the $\pi \rightarrow \pi^{*}$ transition in acrolein (Fig. 2a), the main component of the magnetic-dipole contribution (magnetic field along $z$ ) is invariant to the propagation direction within the $x y$-plane. The observed deviation from cylindrical symmetry can be attributed to the electric-quadrupole-magnetic-dipole cross term that, however, vanishes upon 


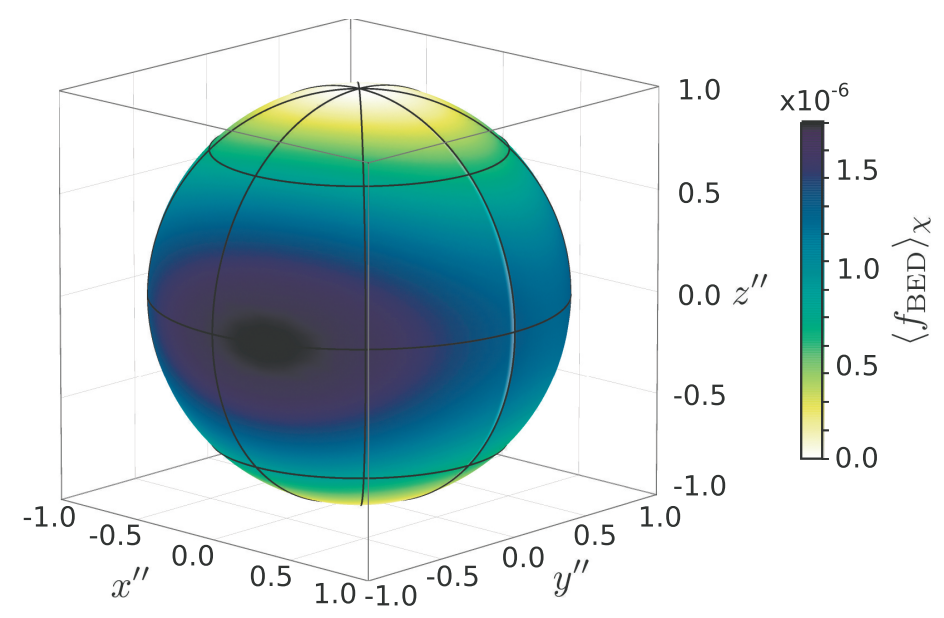

Figure 3. $(\theta, \phi)$-dependency of the $\chi$-averaged oscillator strength $\left\langle f_{\mathrm{BED}}\right\rangle_{\chi}$ for the lowest $n \rightarrow \pi^{*}$ transition in formaldehyde. The orientation of formaldehyde in the molecule-fixed coordinate system is given in Fig. 1.

rotational averaging over the solid angle.[37] Turning to the $n \rightarrow \pi^{*}$ transition in acrolein, the orientational dependence and magnitude of $\langle f\rangle_{\chi}$ reflects the gain in ED intensity due to the broken symmetry of the carbonyl group. The main contribution to the ED component comes from light polarization along the out-of-plane direction, and a close to cylindrical symmetry about the $x$-axis is seen in Fig. 2c. But in the view of Fig. 2d, it is clear that the BED corrections display the features of the corresponding ED forbidden transition in formaldehyde (see Fig. 3).

\section{2. $\quad$ Ferrocene and vinylferrocene}

To demonstrate the behavior of the proposed scheme for X-ray induced transitions that are ED forbidden, we consider the iron near $K$-edge absorption spectra of eclipsed ferrocene $\left(D_{5 h}\right.$ symmetry) and vinylferrocene. These examples have already been studied in detail in Refs. [47] and [50], both experimentally and also theoretically using truncated multipole-expanded expressions for the calculations of oscillator strengths. In Fig. 4, we show the isotropically averaged spectra as determined with use of the CPP formalism both within and beyond the ED approximation. First, we note that our theoretical spectra for the ferrocene derivatives are in good agreement with those reported by Bernadotte et al. based on a full second-order expression for the oscillator strength,[50] and this confirms the validity of the proposed procedure to obtain isotropically averaged linear absorption cross sections beyond the ED approximation.

In ferrocene, the pre-edge peak at $6961.9 \mathrm{eV}$ can be assigned to the ED forbidden transitions from the Fe $1 s$ orbital to the degenerate pair of lowest unoccupied orbitals. These virtual orbitals are composed of the antibonding linear combination of Fe $3 d$-orbitals and the cyclopentadienyl $\pi$-orbitals of $e_{1}^{\prime \prime}$ symmetry. Such transitions are ED forbidden due to symmetry, and the leading term in the linear absorption cross section will therefore come from the electric-quadrupole and magnetic-dipole contributions, as immediately seen from the absence of this peak in the ED spectrum (Fig. 4b).

By introducing the vinyl substituent, the symmetry, and therefore the degeneracy, is lifted, and the $3 d$-orbitals are mixed with the $\pi^{*}$-orbital of the vinyl group. This leads to two distinct pre-edge peaks found at about 6961.9 and $6965.5 \mathrm{eV}$, respectively (see Fig. 4a), at the B3LYP/aug-cc-pVDZ level of theory. The final states contributing to these peaks in vinylferrocene are illustrated by means of isodensity surface plots in Fig. 5. At the B3LYP level, they contain contributions 


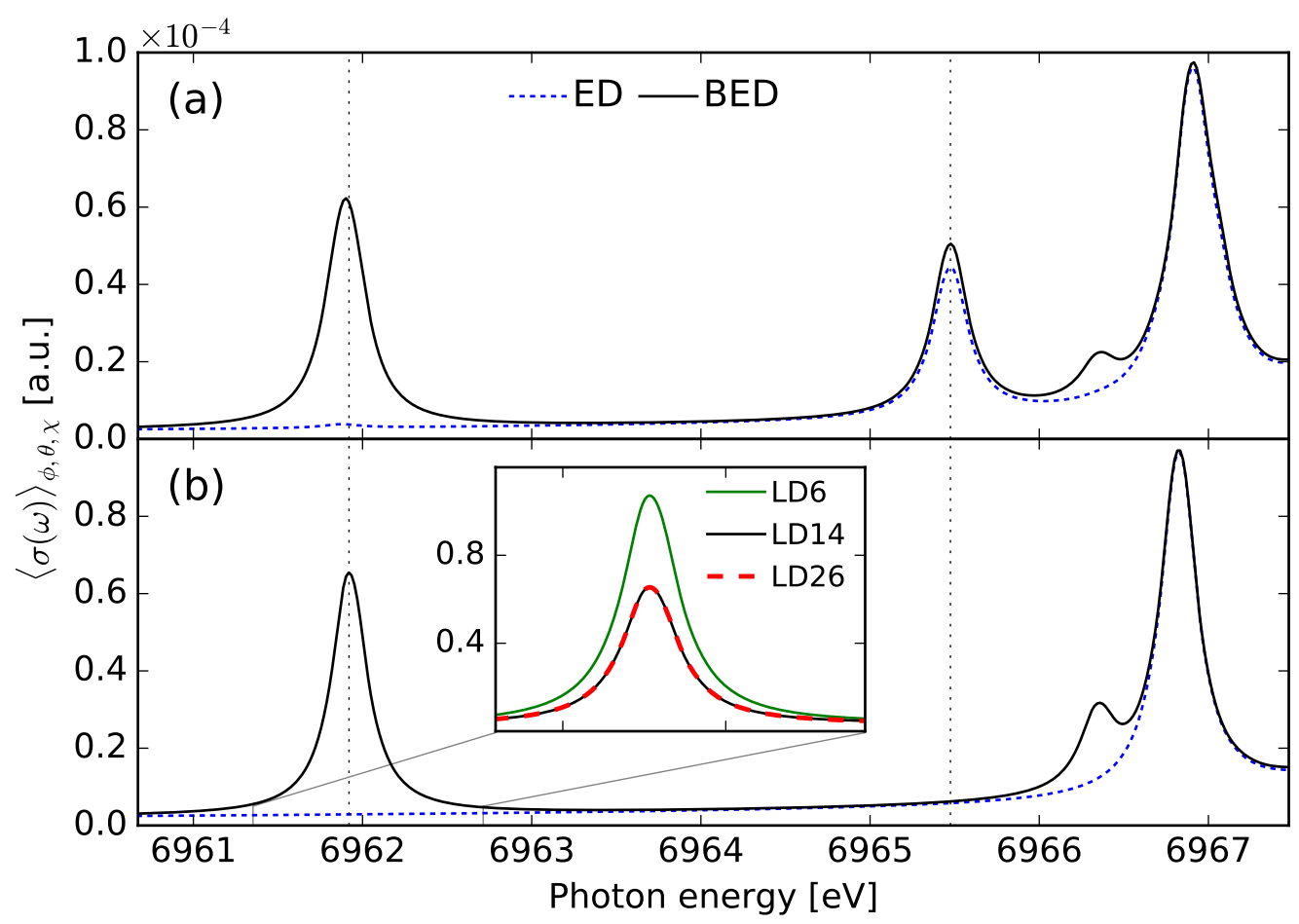

Figure 4. The low-energy part of the isotropically averaged Fe $K$-edge spectra of (a) vinylferrocene and (b) ferrocene from CPP calculations at the B3LYP/aug-cc-pVDZ level of theory as obtained within and beyond the ED approximation, using the LD14 scheme to compute the isotropic average in the latter case. Inset figure shows the convergence of the pre-edge peak of ferrocene with respect to the size of the Lebedev grid (see discussion in Section 4.3). A common lifetime broadening $\gamma=1000 \mathrm{~cm}^{-1}$ has been used.

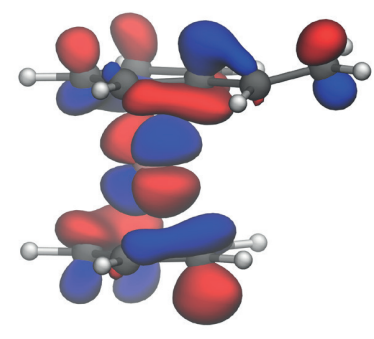

(a)

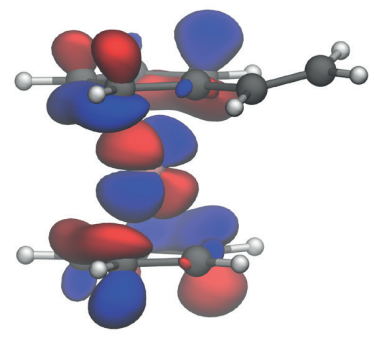

(b)

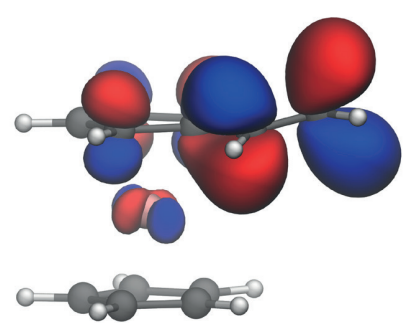

(c)

Figure 5. The final states contributing to the two pre-edge peaks in the Fe $K$-edge spectrum of vinylferrocene: (a) and (b) Fe $d_{x z^{-}}$and $d_{y z}$-orbitals, respectively, underlying the lowest pre-edge peak, and (c) the vinyl $\pi^{*}$-orbital involving significant $\mathrm{Fe} 4 p-3 d$ mixing giving rise to the second pre-edge peak. An isodensity surface value of 0.025 a.u. has been used.

from several single-excited determinants in which the Fe $1 s$-orbital has been replaced by a virtual orbital, and the figure shows the linear combinations of these virtual orbitals. The lowest-energy peak, corresponding to the $1 s \rightarrow 3 d$ transitions discussed above in unsubstituted ferrocene, becomes slightly ED allowed following the vinyl substitution. This can be seen in terms of a very weak feature in the ED spectrum in Fig. 4a (blue dashed curve profile). The second peak originates from a transition from the Fe $1 s$-orbital to a final state dominated by the $\pi^{*}$-orbital of the vinyl substituent, shown in Fig. 5c. However, due to the locality of the Fe $1 s$ orbital, the intensity will be governed by the Fe atomic orbital components of the final state that mainly stem from the $4 p_{z^{-}}$and $3 d_{x z}$-orbitals. This $4 p-3 d$ mixing in the final state explains the relative ED and BED intensity behavior of the second 

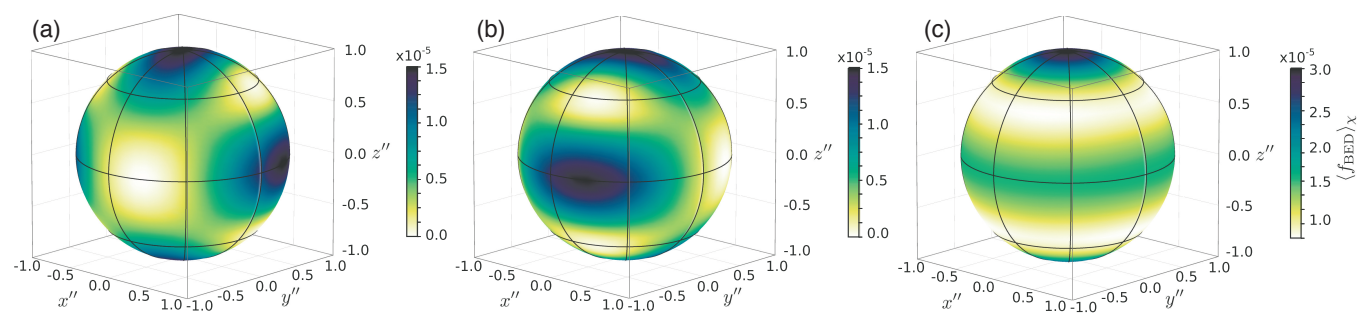

Figure 6. $(\theta, \phi)$-dependency of the $\chi$-averaged oscillator strength $\left\langle f_{\mathrm{BED}}\right\rangle_{\chi}$ for the two transitions underlying the lowest-energy pre-edge peak in the Fe $K$-edge spectrum of vinylferrocene: (a) the $1 s \rightarrow 3 d_{x z}$ transition, (b) $1 s \rightarrow 3 d_{y z}$ transition and (c) the summed contribution from (a) and (b). The orientation of vinylferrocene in the molecular coordinate system is given in Fig. 1.

pre-edge peak, where the ED intensity increases due to the $1 s \rightarrow 4 p$ component of the transition and now exceeds the higher-order contributions arising from the ED forbidden $1 s \rightarrow 3 d$ component, the latter given by the difference between the BED and ED spectra.

The $\chi$-averaged oscillator strength for $1 s \rightarrow 3 d$ transitions shows a stronger dependency on the propagation direction compared to those for the $\pi \rightarrow \pi^{*}$ and $n \rightarrow \pi^{*}$ transitions discussed above. This is illustrated in Figs. 6a and $6 \mathrm{~b}$ for the two transitions giving rise to the lowest-energy pre-peak in the $K$-edge spectrum of vinylferrocene. In these cases, the transition densities are governed primarily by a combination of the very compact Fe $1 s$-orbital and the valence $3 d_{x z^{-}}$and $3 d_{y z^{-}}$ orbitals (Figs. 5a and 5b, respectively). A large transition moment and thereby oscillator strength is thus obtained for a situation with an electric-field polarization along the $z$-direction and propagation along either the $x$ - or $y$-directions in the molecular frame, or vice versa. The effect of the vinyl substituent is small for the first pre-edge peak and $x$ - and $y$-directions are therefore close to identical in this respect. This explains the near-cylindrical symmetry of the summed oscillator strength for the two transitions shown in Fig. 6c. In view of the more complex nodal structure of $\langle f\rangle_{\chi}$ for the $1 s \rightarrow 3 d$ transitions as compared to the corresponding behaviors of the valence and core excitations in formaldehyde and acrolein (Figs. 2 and 3 ), one can anticipate that the numerical integration over angles $(\theta, \phi)$ will be more difficult to converge. This aspect will be discussed in the subsequent section.

\subsection{Convergence rate and computational cost}

Having considered the behavior of the $\chi$-averaged linear absorption cross section, we proceed to investigate the rate of convergence in the numerical integration scheme and compare the computational cost with the corresponding conventional calculations based on truncated multipole expansions.

The isotropically averaged oscillator strengths involving the full field-matter interaction operator for the valence and core transitions in the two aldehydes are listed in Table 1 for progressively larger Lebedev grids $\mathrm{LD} n$, where $n=\{6,14,26,38,50\}$ denotes the number of quadrature points. For comparison, we also include the corresponding oscillator strengths calculated within the ED approximation, and these results have been obtained by means of analytical integration, or, equivalently, tensor averaging.

The number of quadrature points needed when going beyond the ED approximation to integrate the absorption cross section to a certain degree of accuracy is closely associated with the importance of higher-order terms in the wave vector for a given excitation. In general, the higher the order in the expansion that is relevant for the spectrum, the larger the number of sampled orientations required to obtain sufficiently accurate absorption cross sections. This is readily understood from 
the fact that the Lebedev grids are constructed to exactly integrate real spherical harmonics up to a given maximum angular momentum $L_{\max }$ as given in Table 1.[18] For the rotational averaging of the linear absorption cross section, we have to treat a product of two complex conjugate transition moments involving the full field-matter interaction operator. In general, an $m$ 'th rank transition multipole moment $T_{\lambda_{1} \ldots \lambda_{m}}^{[m]}$ in the multipole expansion of the transition moment is expressible as a sum of irreducible tensors of integer orders $l=0,1, \ldots m$ which transform under rotations as spherical harmonics with angular momenta $l$. The product of two transition multipole moments of rank $m_{1}$ and $m_{2}$, as relevant for the linear absorption cross section, will produce a set of irreducible tensors with orders $L$ that lie between $\left|m_{1}-m_{2}\right|$ and $m_{1}+m_{2}$. Accordingly, the smallest LD6 grid $\left(L_{\max }=3\right)$ is sufficient to fully integrate the pure dipole contributions $(L=2)$, and the LD14 scheme $\left(L_{\max }=5\right)$ treats fully the linear absorption through second order in the wave vector.

In comparing results obtained with different number of quadrature points, we can thus identify the character of the contributions to transition intensities in the language of multipole expansions. At the level of LD6, we retrieve in addition to the electric-dipole-electric-dipole $\left(\mu^{2}\right)$ term also the magnetic-dipolemagnetic-dipole $\left(\mathrm{m}^{2}\right)$ term, which transforms under rotation in the same way. The electric-dipole-magnetic-dipole $(\mu \mathrm{m})$ and electric-dipole-electric-quadrupole $(\mu Q)$ cross terms vanish for absorption of linearly polarized light.[37] At the level of LD14, we retrieve, in addition to the contributions mentioned above, also the electric-quadrupole-electric-quadrupole $\left(Q^{2}\right)$ term as well as the electric-dipoleelectric-octupole $(\mu O)$ and electric-dipole-magnetic-quadrupole cross terms $(\mu \mathcal{M})$, which, just like the $m^{2}$ term, is of quadratic order in the wave vector expansion and therefore expected to provide a contribution to the linear absorption cross section that is of similar size.

As seen in Table 1 for formaldehyde and acrolein, the Lebedev quadrature used to rotationally average the oscillator strength converges in general very rapidly, and already at the LD14 level errors are, in all considered cases, within the adopted convergence threshold of $10^{-9}$ for the excitation vectors. The LD6-ED difference in averaged oscillator strengths amounts to $1.2 \times 10^{-6}$ and $2.0 \times 10^{-6}$ for the $n \rightarrow$ $\pi^{*}$ transitions in acrolein and formaldehyde, respectively. These estimates of $m^{2}$ contributions are some three orders of magnitude larger than the $Q^{2}$ contributions (including also $\mu O$ and $\mu \mathcal{M}$ for acrolein) as estimated from the corresponding LD14-LD6 differences. This is in stark contrast to the $1 s \rightarrow 3 d$ transitions in the ferrocene derivatives, where the $m^{2}$ and $Q^{2}$ contributions are of similar size, as illustrated in the inset of Fig. 4b.

Since the inclusion of terms through second order in the wave vector has proven here and elsewhere[10, 37] to provide accurate results for the calculation of linear absorption spectra in both the $\mathrm{UV} /$ vis and X-ray regions of the spectrum, it may be natural to question the sense in turning to a treatment of the full field-matter interaction operator from a computational cost point of view. In the CPP framework, the inclusion of the terms through second order in the wave vector to the complex polarizability in a multipole formulation means that one needs to solve $3+3+6=12$ linear response equations for a given optical frequency, corresponding to the independent components of the multipole moment operators $(\mu, m$ and $Q)$. In the BED framework, on the other hand, the adoption of the LD14 quadrature scheme means that one needs to solve $2 \times 7=14$ linear response equations for a given optical frequency, corresponding to the seven independent wave vector points and the two components of the linear momentum operator $\left(\hat{p}_{x}^{\prime}\right.$ and $\left.\hat{p}_{y}^{\prime}\right)$. So the conclusion is that, not only does the LD14 scheme provide a highly accurate 
level of numerical integration of the BED absorption cross section (with, amongst others, its virtue of gauge-origin independence) but it is on par with a conventional multipole expansion scheme in terms of computational efficiency.

\section{Conclusion}

We have presented a scheme to perform rotational averaging of linear absorption cross sections involving the full field-matter interaction operator. Our approach is based on a combination of numerical and analytical integration over the three Euler angles. The former is performed with use of Lebedev quadratures over the solid angle and corresponds to integration over all possible directions of the wave vector of the electromagnetic field, and the latter to averaging over the associated polarization vector. The computational cost to accomplish this achievement is not significantly higher than what is found in response theory calculations based on conventional multipole expansions.

This work ensures the calculation of gauge-origin independent and positive linear absorption cross sections in isotropic samples, also beyond the ED approximation. This is particularly important in cases where the magnetic-dipole and electricquadrupole contributions to the cross section are larger than or comparable to the ED contribution, such as in $n \rightarrow \pi^{*}$ transitions in organic compounds and $K$-edge spectra of transition metal complexes.

\section{Acknowledgements}

It is our great pleasure to dedicate this paper to the 60th anniversary of Prof. Hans Jørgen Aa. Jensen. His outstanding scientific contributions in general and his long-term commitment to the Dalton and DIRAC projects in particular are both of great value to us all. We are very grateful for his sharing of knowledge and scientific guidance as well as being a dear friend.

The authors thank Prof. Christoph Jacob for kindly providing the structures of ferrocene and vinylferrocene. N. H. L. acknowledges the Carlsberg Foundation for a postdoctoral fellowship. P. N. acknowledges financial support from Knut and Alice Wallenberg Foundation (Grant No. KAW-2013.0020), and the Swedish Research Council (Grant No. 621-2014-4646). Computational resources provided by the Swedish National Infrastructure for Computing (SNIC) at National Supercomputer Centre (NSC), Sweden, are acknowledged.

\section{References}

[1] J. Kagan, Organic Photochemistry: Principles and Applications (Elsevier Science, Chichester, 2012).

[2] G. Zomer, J.W. Hastings, F. Berthold, A. Lundin, A.M. Garcia Campana, R. Niessner, T.K. Christopolous, C. Lowik, B. Branchini, S. Daunert, L. Blum and L.J. Kricka, Chemiluminescence and Bioluminescence (The Royal Society of Chemistry, Cambridge, 2011).

[3] H.S. Nalwa, editor, Handbook of Advanced Electronic and Photonic Materials and Devices (Academic Press, San Diego, 2001).

[4] J.C. Lindon, G.E. Tranter and J.L. Holmes, editors, Encyclopedia of Spectroscopy and Spectrometry (Academic Press, San Diego, 2010).

[5] H. Shirani, M. Linares, C. Sigurdson, M. Lindgren, P. Norman and K. Nilsson, Chem. Eur. J. 21, 15133 (2015).

[6] P. Norman, Phys. Chem. Chem. Phys. 13, 20519 (2011). 
[7] S. Mukamel, Principles of Nonlinear Optical Spectroscopy, Vol. 29 (Oxford University Press New York, New York, 1995).

[8] L.D. Barron, Molecular Light Scattering and Optical Activity (Cambridge University Press, Cambridge, 2004).

[9] R.W. Boyd, Nonlinear Optics (Academic press, Boston, 2003).

[10] N.H. List, J. Kauczor, T. Saue, H.J.Aa. Jensen and P. Norman, J. Chem. Phys. 142 (24), $244111(2015)$.

[11] J. Stöhr, NEXAFS Spectroscopy, Vol. 25 (Springer, Berlin, 1992).

[12] D.H. Friese, M.T.P. Beerepoot and K. Ruud, J. Chem. Phys. 141, 204103 (2014).

[13] G. Wagnière, J. Chem. Phys. 76 (1), 473 (1982).

[14] D. Andrews and W. Ghoul, J. Phys. A Math. Gen. 14 (6), 1281 (1981).

[15] D. Andrews and T. Thirunamachandran, J. Chem. Phys. 67 (11), 5026 (1977).

[16] D.L. Andrews and N.P. Blake, J. Phys. A Math. Gen. 22 (1), 49 (1989).

[17] S.S. Andrews, J. Chem. Educ. 81 (6), 877 (2004).

[18] V.I. Lebedev and D.N. Laikov, Dokl. Math. 59, 477 (1999), Angular quadrature parameters available from: http://server.ccl.net/cca/software/SOURCES/FORTRAN/ Lebedev-Laikov-Grids/index.shtml.

[19] V.I. Lebedev, Russ. Acad. Sci. Dokl. Math. 50 (2), 283 (1995).

[20] V.I. Lebedev and A.L. Skorokhodov, Russ. Acad. Sci. Dokl. Math. 45 (2), 587 (1992).

[21] V.I. Lebedev, Sib. Math. J. 18 (1), 99 (1977).

[22] V.I. Lebedev, USSR Comput. Math. Math. Phys. 16 (2), 10 (1976).

[23] V.I. Lebedev, USSR Comput. Math. Math. Phys. 15 (1), 44 (1975).

[24] G.C. Schatz and M.A. Ratner, Quantum Mechanics in Chemistry (Courier Corporation, Oxford, 2002).

[25] H. Mahr, Two-Photon Absorption Spectroscopy, in Quantum Electronics - Nonlinear Optics, Part A, edited by H. Rabin and C. L. Tang, Vol. 1 (Academic Press, New York, 1975).

[26] P. Norman, D.M. Bishop, H.J.Aa. Jensen and J. Oddershede, J. Chem. Phys. 115 (22), 10323 (2001).

[27] P. Norman, D.M. Bishop, H.J.Aa. Jensen and J. Oddershede, J. Chem. Phys. 123 (19), 194103 (2005).

[28] U. Ekström and P. Norman, Phys. Rev. A 74, 042722 (2006).

[29] E. Merzbacher, Quantum Mechanics (Wiley, Chichester, 1970).

[30] B.H. Bransden and C.J. Joachain, Physics of Atoms and Molecules, 2nd ed. (England New York Prentice Hall, Harlow, 2003).

[31] T. Yamamoto, X-Ray Spectrom. 37 (6), 572 (2008).

[32] G. Shulman, Y. Yafet, P. Eisenberger and W. Blumberg, Proc. Natl. Acad. Sci. U.S.A. 73 (5), 1384 (1976).

[33] J.E. Hahn, R.A. Scott, K.O. Hodgson, S. Doniach, S.R. Desjardins and E.I. Solomon, Chem. Phys. Lett. 88 (6), 595 (1982).

[34] S.D. George, T. Petrenko and F. Neese, Inorg. Chim. Acta 361 (4), 965 (2008).

[35] L.N. Vidal, F. Egidi, V. Barone and C. Cappelli, J. Chem. Phys. 142 (17), 174101 (2015).

[36] M. Anelli, D. Jonsson, H. Fliegl and K. Ruud, Mol. Phys. 113 (13-14), 1899 (2015).

[37] S. Bernadotte, A.J. Atkins and C.R. Jacob, J. Chem. Phys. 137 (20), 204106 (2012).

[38] P.J. Lestrange, F. Egidi and X. Li, J. Chem. Phys. 143 (23), 234103 (2015).

[39] S.L. Altmann, Rotations, Quaternions, and Double Groups (Dover Publications, Mineola, New York, 2005).

[40] P.J. Stephens, F.J. Devlin, C.F. Chabalowski and M.J. Frisch, J. Phys. Chem. 98 (46), 11623 (1994).

[41] A.D. Becke, J. Chem. Phys. 98 (7), 5648 (1993).

[42] S.H. Vosko, L. Wilk and M. Nusair, Can. J. Phys. 58 (8), 1200 (1980).

[43] C. Lee, W. Yang and R.G. Parr, Phys. Rev. B 37, 785 (1988).

[44] T.H. Dunning, J. Chem. Phys. 90 (2), 1007 (1989).

[45] T. Yanai, D.P. Tew and N.C. Handy, Chem. Phys. Lett. 393 (1-3), 51 (2004).

[46] M. Stener, G. Fronzoni and M. De Simone, Chem. Phys. Lett. 373 (1), 115 (2003).

[47] A.J. Atkins, C.R. Jacob and M. Bauer, Chem. Eur. J. 18 (23), 7021 (2012).

[48] J. Kauczor, P. Jørgensen and P. Norman, J. Chem. Theory Comput. 7 (6), 1610 (2011).

[49] J. Kauczor and P. Norman, J. Chem. Theory Comput. 10 (6), 2449 (2014).

[50] A.J. Atkins, M. Bauer and C.R. Jacob, Phys. Chem. Chem. Phys. 15 (21), 8095 (2013).

[51] C.v. Wüllen (Private communication), see also http://server.ccl.net/cgi-bin/ccl/ message.cgi?2000+03+03+003+raw.

[52] R.A. Kendall, T.H. Dunning Jr. and R.J. Harrison, J. Chem. Phys. 96 (9), 6796 (1992).

[53] N.B. Balabanov and K.A. Peterson, J. Chem. Phys. 123 (6), 064107 (2005). 
[54] K. Aidas, C. Angeli, K.L. Bak, V. Bakken, R. Bast, L. Boman, O. Christiansen, R. Cimiraglia, S. Coriani, P. Dahle, E.K. Dalskov, U. Ekström, T. Enevoldsen, J.J. Eriksen, P. Ettenhuber, B. Fernández, L. Ferrighi, H. Fliegl, L. Frediani, K. Hald, A. Halkier, C. Hättig, H. Heiberg, T. Helgaker, A.C. Hennum, H. Hettema, E. Hjertenæs, S. Høst, I.M. Høyvik, M.F. Iozzi, B. Jansik, H.J.Aa. Jensen, D. Jonsson, P. Jørgensen, J. Kauczor, S. Kirpekar, T. Kjærgaard, W. Klopper, S. Knecht, R. Kobayashi, H. Koch, J. Kongsted, A. Krapp, K. Kristensen, A. Ligabue, O.B. Lutnæs, J.I. Melo, K.V. Mikkelsen, R.H. Myhre, C. Neiss, C.B. Nielsen, P. Norman, J. Olsen, J.M.H. Olsen, A. Osted, M.J. Packer, F. Pawlowski, T.B. Pedersen, P.F. Provasi, S. Reine, Z. Rinkevicius, T.A. Ruden, K. Ruud, V. Rybkin, P. Sałek, C.C.M. Samson, A.S. de Merás, T. Saue, S.P.A. Sauer, B. Schimmelpfennig, K. Sneskov, A.H. Steindal, K.O. Sylvester-Hvid, P.R. Taylor, A.M. Teale, E.I. Tellgren, D.P. Tew, A.J. Thorvaldsen, L. Thøgersen, O. Vahtras, M.A. Watson, D.J.D. Wilson, M. Ziolkowski and H. Agren, WIREs Comput. Mol. Sci. 4 (3), 269 (2014).

[55] Dalton, A Molecular Electronic Structure Program, Release DALTON2015.0 (2015) (http: //daltonprogram.org/). 
